\title{
The Search for Secreted Proteins in Prostate Cancer by the Escherichia coli Ampicillin Secretion Trap: Expression of NBL1 Is Highly Restricted to the Prostate and Is Related to Cancer Progression
}

\author{
Tetsutaro Hayashi ${ }^{a, b}$ Kazuhiro Sentani ${ }^{a}$ Naohide Oue $^{a}$ Shinya Ohara ${ }^{b}$ \\ Jun Teishima ${ }^{b}$ Katsuhiro Anami ${ }^{a}$ Naoya Sakamoto ${ }^{a}$ Akio Matsubara ${ }^{b}$ \\ Wataru Yasuia \\ Departments of a Molecular Pathology and b ${ }^{b}$ rology, Hiroshima University Graduate School of Biomedical \\ Sciences, Hiroshima, Japan
}

\section{Key Words}

CAST $\cdot$ NBL1 $\cdot$ Prostate cancer

\begin{abstract}
Aims: Genes expressed only in cancer tissue or specific organs will be useful molecular markers. To identify genes that encode secreted proteins present in prostate cancer (PCa), we generated Escherichia coli ampicillin secretion trap (CAST) libraries from PCa and normal prostate (NP). Methods and Results: We identified 15 candidate genes that encode secreted proteins present in PCa and NP. Quantitative RT-PCR analysis revealed that $M S M B, N B L 1$ and $A Z G P 1$ were expressed with much higher specificity in PCa and NP than in 14 other kinds of normal tissue. We focused on NBL1, which was originally identified as a putative tumor suppressor gene. Western blot analysis revealed that NBL1 protein was highly expressed in both cell lysate and culture media of the DU145 PCa cell line. Immunohistochemical analysis showed that NBL1 expression was highly detected in and restricted to NP and PCa and was significantly down-regulated in PCa. NBL1 expression was significantly reduced according to the tumor stage, Gleason grade and preoperative prostate-specific antigen (PSA) value. Conclusion: NBL1 is a secreted pro-
\end{abstract}

tein that is highly restricted to the prostate. Underexpression of NBL1 correlated with PCa progression. NBL1 might be a candidate tumor marker for PCa in addition to PSA.

Copyright $\odot 2012$ S. Karger AG, Basel

\section{Introduction}

Prostate cancer $(\mathrm{PCa})$ is one of the most common human male cancers. Cancer develops as a result of multiple genetic and epigenetic alterations [1]. Better knowledge of changes in gene expression that occur during prostatic carcinogenesis may lead to improvements in its diagnosis, treatment and prevention [2]. Genes encoding secretory proteins expressed specifically in cancers or specific organs may be ideal biomarkers for cancer diagnosis. To identify novel genes that encode secreted protein present in PCa, we used the Escherichia coli ampicillin secretion trap (CAST) method. We have previously identified several PCa-specific genes encoding transmembrane proteins with the CAST method and reported their high potential as therapeutic targets $[3,4]$. To our knowledge, however, CAST analysis of secreted protein in PCa has not been reported.

\section{KARGER}

Fax +4161306 1234

E-Mail karger@karger.ch

www.karger.com
(C) 2012 S. Karger AG, Basel

1015-2008/13/0802-0060\$38.00/0

Accessible online at:

www.karger.com/pat
Dr. Wataru Yasui

Department of Molecular Pathology

Hiroshima University Graduate School of Biomedical Sciences

1-2-3 Kasumi, Minami-ku, Hiroshima 734-8551 (Japan)

Tel. +81 82257 5145, E-Mail wyasui@ hiroshima-u.ac.jp 
Prostate-specific antigen (PSA), a serine protease found in semen, is the most widely used serum marker for detecting and monitoring PCa $[5,6]$. The rapid incorporation of aggressive PSA testing has resulted in dramatically earlier identification of $\mathrm{PCa}$ and is attributed with the decrease in mortality from PCa [7]. However, there are limitations to the use of PSA. PSA levels are also increased in benign prostatic hyperplasia $(\mathrm{BPH})$ and general inflammatory responses. PSA testing has the potential disadvantage of low specificity and has led to a tremendous increase in the number of unnecessary prostate biopsies [8]. Furthermore, blood PSA level is not significantly increased in patients with poorly differentiated $\mathrm{PCa}$ and patients receiving androgen deprivation therapy $[9,10]$. Moreover, the prognosis of castration-resistant PCa remains unsatisfactory [11]. Therefore, increasing emphasis has been placed on the need to determine new protein biomarkers for use in the diagnosis of PCa.

In the present study, to identify genes that encode secreted proteins, we generated CAST libraries from $2 \mathrm{PCa}$ cell lines, LNCaP and DU145, and normal prostate (NP). CAST is a signal sequence trap method developed by Ferguson et al. [12]. Signal peptides target secreted and transmembrane proteins to their appropriate subcellular localization [13]. A consensus sequence for the signal peptide has not been identified, and, thus, standard molecular techniques are not well suited to identify such proteins. CAST exploits the ability of mammalian signal sequences to confer ampicillin resistance to a mutant $\beta$-lactamase lacking the endogenous signal sequence [14]. We report here the identification of several genes that encode secreted proteins expressed in PCa and NP. Among these, we focused on the NBL1 gene because this gene is highly restricted in PCa and NP. NBL1 (neuroblastoma suppression of tumorigenicity 1) was originally identified as a putative tumor suppressor gene in a transformed fibroblast rat model [15]. We confirmed the expression of NBL1 in cell lysate and culture media of PCa cells. We examined the expression and distribution of NBL1 in human PCa and NP by immunohistochemistry and compared them with clinicopathological characteristics. We also studied the expression of NBL1 in normal systemic organs because NBL1 has the possibility of being a serum marker for PCa.

\section{Materials and Methods}

\section{CAST Library Construction}

Plasmid CAST (pCAST) was designed to contain the kanamycin resistance gene and the $\beta$-lactamase gene lacking the first 69 nucleotides encoding the endogenous signal peptide. EcoRI and
Table 1. Characteristics of the PCa patients $(n=181)$

\begin{tabular}{llr}
\hline Age & $<70$ years & $92(51 \%)$ \\
& $\geq 70$ years & $89(49 \%)$ \\
PSA at diagnosis & $<20$ & $108(60 \%)$ \\
& 20 to $<100$ & $31(17 \%)$ \\
Gleason score & $\geq 100$ & $42(23 \%)$ \\
& 6 & $64(35 \%)$ \\
pStage & 7 & $44(24 \%)$ \\
& $8-10$ & $40(22 \%)$ \\
Treatment & $\mathrm{B}$ & $33(18 \%)$ \\
& $\mathrm{C}$ & $57(31 \%)$ \\
& $\mathrm{D}$ & $79(44 \%)$ \\
& prostatectomy & $45(25 \%)$ \\
& hormone and/or radiation & $127(70 \%)$ \\
& & $54(30 \%)$ \\
\hline
\end{tabular}

Bam HI sites were placed upstream of the mutant $\beta$-lactamase gene for directional cloning. CAST library construction was performed as described previously $[3,12]$. In brief, CAST cDNA libraries were generated from $2 \mu \mathrm{g}$ of mRNA with a random primer containing a BamHI restriction site for reverse transcription (SuperScript Choice System; Invitrogen, Carlsbad, Calif., USA). The EcoRI-adapted cDNA was digested with BamHI, size fractionated, ligated into pCAST and plated onto Luria-Bertani/ampicillin medium. Individual colonies were picked and grown in $1.0 \mathrm{ml}$ of this medium with kanamycin in a 96-well format. Plasmid DNA was sequenced in a 96-well format using a primer located within the $\beta$-lactamase gene.

\section{Tissue Samples}

In total, 219 primary tumor samples were collected from patients diagnosed with PCa and those with NP. Patients were treated at the Hiroshima University Hospital or an affiliated hospital. Because written informed consent was not obtained, for strict privacy protection, identifying information for all samples was removed before analysis. This procedure was in accordance with the Ethical Guidelines for Human Genome/Gene Research of the Japanese Government.

For quantitative RT-PCR, 16 PCa samples and 9 non-neoplastic samples were used. Samples were frozen immediately in liquid nitrogen and stored at $-80^{\circ} \mathrm{C}$ until use. We confirmed microscopically that the tumor specimens consisted mainly $(>50 \%)$ of cancer cells. Noncancerous samples of heart, lung, stomach, small intestine, colon, liver, pancreas, kidney, bone marrow, peripheral leukocytes, spleen, skeletal muscle, brain and spinal cord were purchased from Clontech (Palo Alto, Calif., USA).

For immunohistochemical analysis, we used archival formalinfixed, paraffin-embedded tissues from 127 PCa patients treated by radical prostatectomy, $54 \mathrm{PCa}$ patients who underwent prostate biopsy, and 13 patients treated by suprapubic prostatectomy for $\mathrm{BPH}$. Tumor staging was in accordance with the TNM classification system [16], and histological classification of PCa was made in accordance with the World Health Organization classification [17]. The clinical characteristics of the PCa patients are shown in table 1 . In addition, we used archival formalin-fixed, paraffin-embedded tissues from normal systemic organs including 5 speci- 
Table 2. List of genes encoding secreted protein from the CAST libraries

Sample name

LNCaP DU145 Normal

Prostate

\begin{tabular}{llll}
\hline $\begin{array}{c}\text { Genes encoding secreted } \\
\text { protein }\end{array}$ & \multicolumn{1}{c}{6} & \multicolumn{1}{c}{8} & \multicolumn{1}{c}{7} \\
\hline Gene name & TFPI & TFPI & MSMB \\
& SPP1 & CCDC126 & AZGP1 \\
& FN1 & CLU & SPP1 \\
& COL4A5 & DMKN & TFPI \\
& CALU & NBL1 & C1RL \\
& SFTPA1B & ARMETL & CLU \\
& & FGB & SRGN \\
& & NTN4 & \\
\hline
\end{tabular}

mens each of the brain, spinal cord, heart, lung, esophagus, stomach, small intestine, colon, liver, spleen, pancreas, kidney, adrenal, ureter, bladder, testis, skin, skeletal muscle and blood vessels.

\section{Quantitative RT-PCR and Western Blot Analysis}

Quantitative RT-PCR was performed with an ABI PRISM 7700 sequence detection system (Applied Biosystems, Foster City, Calif., USA) as described previously [18]. Quantitation of NBL1 mRNA levels was done by real-time fluorescence detection as reported in a previous study [19]. The NBL1 primer sequences were 5'-TCAACAAGCTGGCACTGTTC-3' and 5'-GCAGGAGTCACAGTGAACCA-3' (for more information, see online suppl. material at www.karger.com/doi/10.1159/000341396).

For Western blot analysis, tissue samples or cells were lysed as described previously [20]. The primary antibody against NBL1 (R\&D Systems, Inc., Minneapolis, Minn., USA) was used (see online suppl. material).

\section{Evaluation of Specificity of Gene Expression}

To evaluate the specificity of expression in each gene, a specificity index was calculated as follows: first, we identified the 14 normal tissues, which are indispensable for survival, in which the target gene expression was highest among tissues analyzed by quantitative RT-PCR. We then identified PCa among the 16 PCa samples in which the target gene expression was highest by quantitative RT-PCR (mRNA expression level in this tissue was denoted as A). We next identified NP among the 9 NP samples in which the target gene expression was highest by quantitative RT-PCR (mRNA expression level in this tissue was denoted as B). The target mRNA expression levels were standardized to normal organ with highest expression set as 1 . A and B were defined as the PCa and NP specificity indices, respectively. When the specificity index of the target gene in A and/or B was $\geq 10$, the gene was considered to show high specificity. When the specificity index of the target gene was $<10$ and $\geq 5$, the gene was considered to show low specificity. When the specificity index of the target gene was $<5$, the gene was considered to show no specificity.

\section{Immunohistochemistry}

Immunohistochemical analysis was performed with a Dako Envision+ mouse peroxidase detection system (Dako Cytomation, Carpinteria, Calif., USA). The following antibody dilutions were used: goat polyclonal anti-NBL1, 1:100 (R\&D Systems; see supplementary material). We used the percentage of NBL1-positive cells in the total cells as an immunohistochemical scoring system. Evaluation of immunoreactivity was scored independently by two pathologists (K.S. and T.H.). The sections from the 127 patients treated by radical prostatectomy were evaluated in both cancerous and non-cancerous areas. The sections from the 54 prostate biopsy patients were evaluated in the cancerous area. The percentage of NBL1 expression in each region was scored as follows: no expression, $0 ;<10 \%, 1+; 10-30 \%, 2+; 31-50 \%, 3+$, and $>50 \%, 4+$. We used NBL1 expression in nerve cells as a positive control based on previously reported NBL1 immunoreactivity in nerve cells [21].

\section{RNA Interference and Cell Growth and in vitro Invasion} Assays

RNA interference was performed to knock down the endogenous NBL1. siRNA oligonucleotides for NBL1 and a negative control were purchased from Invitrogen (Carlsbad, Calif., USA). The MTT [3-(4,5-dimethylthiazol-2-yl)-2,5-diphenyltetrazolium bromide] and modified Boyden chamber assays were performed to examine cell growth and invasiveness, respectively (see online suppl. material) [22, 23].

\section{Statistical Method}

Associations between clinicopathological variables and NBL1 expression were analyzed by Mann-Whitney U test. The comparison of cell growth and invasive activity was analyzed by t test. A value of $\mathrm{p}<0.05$ was considered statistically significant.

\section{Results}

\section{Identification of Genes with Higher Expression in the Prostate than in 14 Normal Tissues}

To identify genes that encode secreted proteins present in $\mathrm{PCa}$, we generated CAST libraries from $2 \mathrm{PCa}$ cell lines (LNCaP and DU145) and NP, as previously described [3, 4]. We identified 6, 7 and 8 genes encoding secreted proteins from the respective cell lines and NP. The names of these 21 genes are shown in table 2 . We performed quantitative RT-PCR to identify genes expressed specifically in PCa and NP. Representative results are shown in figure 1. We then identified the PCa among 16 tissues in which the target gene expression was highest (mRNA expression levels are shown as A; table 3 ) and NP among 9 tissues in which the target gene expression was highest (mRNA expression levels are shown as $\mathrm{B}$; table 3 ). Next, the $\mathrm{PCa}$ specificity index (A/B ratio) for each gene was calculated. We could not find a gene specific only to $\mathrm{PCa}$, but we could detect genes specific to both PCa and NP. Because 


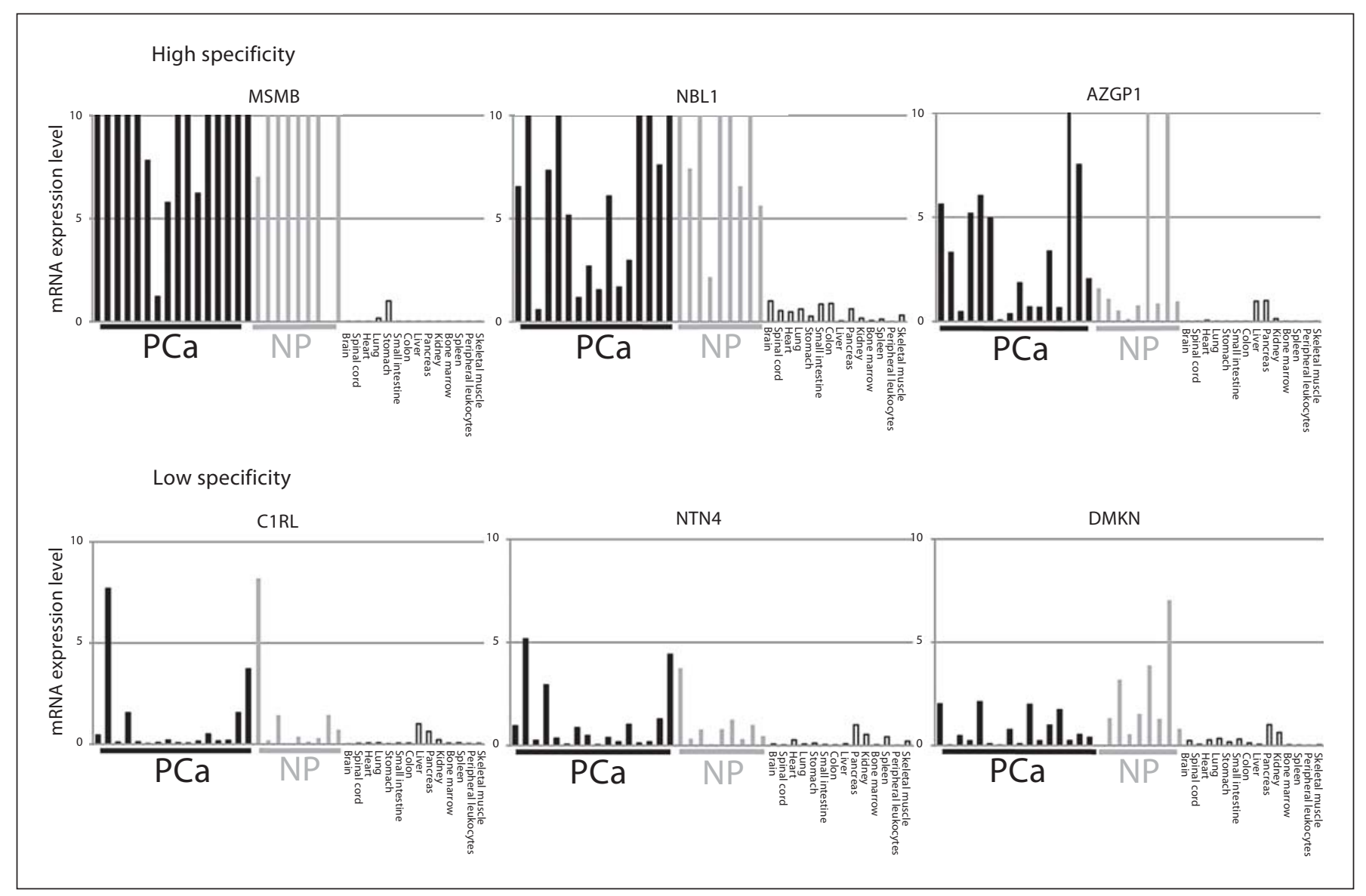

Fig. 1. Quantitative RT-PCR analysis of candidate genes in 16 PCa samples, 9 NP samples, and 14 kinds of normal tissues. MSMB, NBL1 and AZGP1 were found to show high specificity for the prostate. C1RL, NTN4 and DMKN were found to show low specificity for the prostate.

PSA is also specific for prostate and not specific for $\mathrm{PCa}$, we examined these prostate-specific genes. Of the 15 candidates, 3 genes, $M S M B, N B L 1$ and $A Z G P 1$, were found to show high specificity for the prostate, and 3 genes, $C 1 R L$, NTN4 and DMKN, were found to show low specificity for the prostate. Of the 3 genes showing high specificity, MSMB (microseminoprotein- $\beta$ ) and AZGP1 (zinc- $\alpha_{2^{-}}$ glycoprotein) have already been studied in PCa, and their utility as serum tumor marker and therapeutic target have been reported [24-27]. Therefore, we focused on NBL1. NBL1 has been reported as one of the genes whose expression is reduced in $\mathrm{PCa}$ compared with NP by profiling of expressed sequence tags and quantitative RTPCR analysis $[28,29]$. However, there is no report, to our knowledge, in which NBL1 expression in human systemic organs is compared and NBL1 expression in PCa and NP determined by immunohistochemical analysis has not been reported.

CAST Analysis of Secreted Protein in $\mathrm{PCa}$
We compared NBL1 mRNA expression between prostate and systemic normal organs. The average NBL1 mRNA expression levels in NP and PCa were 19.9- and 8.1-fold greater, respectively, than in brain, in which NBL1 is most highly expressed of the systemic normal organs. In comparison with that in the other systemic organs, the expression of NBL1 mRNA is highly restricted to the prostate.

\section{NBL1 Protein Expression in Cell Lysate and Culture Medium}

To study whether NBL1 is a secreted protein, we performed Western blot analysis in 3 PCa cell lines. In cell lysate, moderate NBL1 expression was detected in DU145 cells as a band of approximately $27 \mathrm{kDa}$. LNCaP cells showed low NBL1 expression, and NBL1 expression was absent in PC3 cells (fig. 2a). In culture medium, very high NBL1 expression was noted in DU145 cells, low NBL1 ex- 
Table 3. Summary of quantitative RT-PCR analysis of candidate genes specifically expressed in PCa and NP samples

\begin{tabular}{lllllll}
\hline Gene name & $\begin{array}{l}\text { Name of normal } \\
\text { organ with highest } \\
\text { expression }\end{array}$ & $\begin{array}{l}\text { PCa with } \\
\text { highest mRNA } \\
\text { expression level } \\
\text { (A) }\end{array}$ & $\begin{array}{l}\text { NP with } \\
\text { highest mRNA } \\
\text { expression level } \\
\text { (B) }\end{array}$ & $\begin{array}{l}\text { PCa } \\
\text { specificity } \\
\text { index } \\
(\mathrm{A} / \mathrm{B})\end{array}$ & $\begin{array}{l}\text { PCa cases with } \\
\text { mRNA expression } \\
\text { level } \geq 10 \text {-fold } \\
\text { normal organ with } \\
\text { highest expression }\end{array}$ & $\begin{array}{l}\text { NP cases with } \\
\text { mRevel } \geq 10 \text {-fold } \\
\text { normal organ with } \\
\text { highest expression }\end{array}$ \\
\hline High specificity & & & & & \\
MSMB & Stomach & $>100$ & $>100$ & 0.13 & 12 & 8 \\
NBL1 & Brain & 29.6 & 71.2 & 0.42 & 5 & 5 \\
AZGP1 & Pancreas & 21.5 & 22.4 & 0.96 & 1 & 2 \\
\hline Low specificity & & & & & 0 \\
C1RL & Liver & 7.7 & 8.2 & 0.94 & 0 & 0 \\
NTN4 & Pancreas & 5.2 & 3.7 & 1.4 & 0 & 0 \\
DMKN & Pancreas & 2.1 & 7.0 & 0.3 & 0 & 0 \\
\hline No specificity & Liver & & & & & 0 \\
FN1 & Stomach & 0.78 & 0.37 & 2.1 & 0 & 0 \\
COL4A5 & 0.72 & 0.69 & 1.0 & 0 & 0 \\
CCDC126 & Skeletal muscle & 0.58 & 0.07 & 8.3 & 0 & 0 \\
SPP1 & Kidney & 0.56 & 0.34 & 1.6 & 0 & 0 \\
CALU & Heart & 0.32 & 0.26 & 1.2 & 0 & 0 \\
CLU & Liver & 0.3 & 1.0 & 0.3 & 0 & 0 \\
SRGN & Bone marrow & 0.27 & 0.67 & 0.4 & 0 & 0 \\
FGB & Liver & $<0.01$ & $<0.01$ & - & 0 & 0 \\
TFPI & Kidney & $<0.01$ & 0.022 & - & 0 & 0 \\
SFTPA1B & Lung & 0.057 & $<0.01$ & - & 0 & 0 \\
ARMETL & Skeletal muscle & $<0.01$ & $<0.01$ & - & 0 & 0 \\
\hline
\end{tabular}

Target mRNA expression levels were standardized to $1.0 \mu \mathrm{g}$ total RNA from the normal organ with the highest expression set as 1.0 (16 PCa and 9 NP samples).

pression was seen in LNCaP cells, and no expression of NBL1 was noted in PC 3 cells. In the CAST analysis, colonies containing the NBL1 gene were detected in the DU145 CAST library, indicating that Western blot analysis of NBL1 protein was consistent with CAST analysis. Next, we examined transition of NBL1 expression by Western blot analysis of cell extracts of DU145 transfected with NBL1-specific siRNAs. Two types of siRNAs (siRNA1 and siRNA2) and negative control siRNA were transfected into DU145 cell extracts. Expression of NBL1 in DU145 was suppressed by treatment with siRNA1 and siRNA2 in both cell lysate and culture medium (fig. 2b).

\section{Immunohistochemical Analysis of NBL1 Expression in Normal Systemic Organs}

We performed immunohistochemical analysis of NBL1 in 19 kinds of non-cancerous systemic tissues in 5 samples of each tissue. NBL1 expression was detected only in epithelium of the small intestine and colon, islets of the pancreas, and nerve cells in brain and spinal cord (fig. 3ad). We did not detect the expression of NBL1 in heart, lung, esophagus, stomach, liver, spleen, pancreas, kidney, adrenal, ureter, bladder, testis, skin, skeletal muscle and vessel. When the level of NBL1 expression is compared in small intestine and colon, islets of the pancreas, and nerve cells in brain and spinal cord, expression in the brain nerve cells is higher than in the other tissues. These results are consistent with our quantitative RT-PCR results.

\section{Immunohistochemical Analysis of NBL1 Expression in $P C a$ and NP}

We also performed immunohistochemical analysis of NBL1 in a total of 194 prostate samples, which included $\mathrm{BPH}$ in 13 patients and $\mathrm{PCa}$ in 181 patients. The clinical characteristics of the PCa patients are summarized in table 1. NBL1 staining was observed in the cytoplasm of both NP epithelium and cancer cells. In some acini, NBL1 expression was stronger at the apical side of luminal cells 


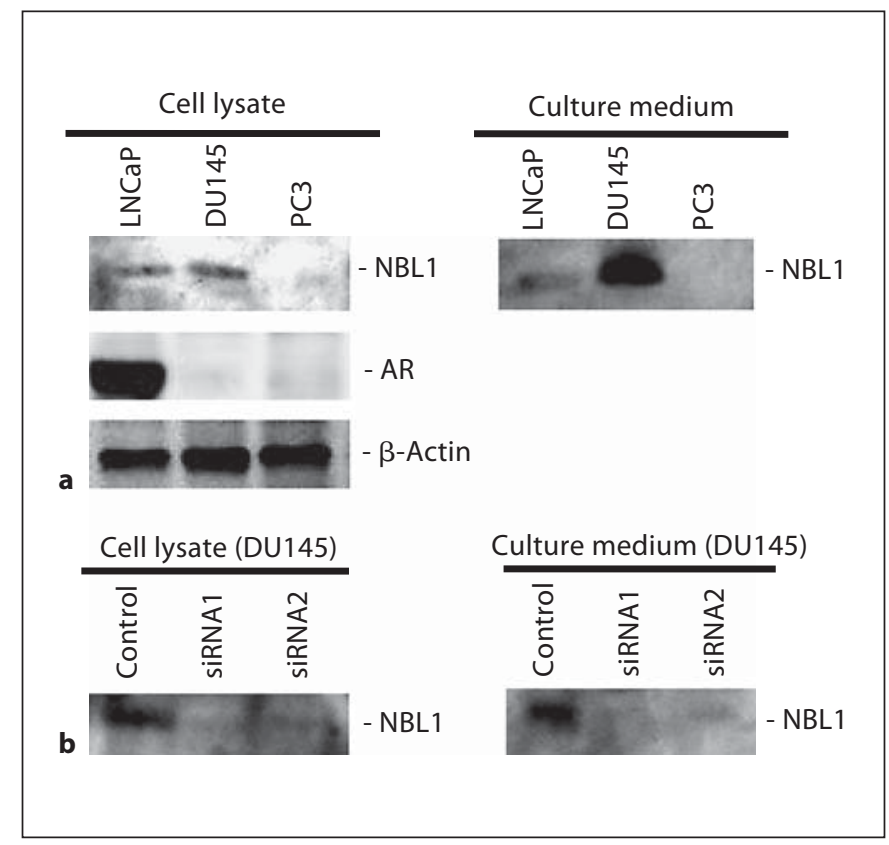

Fig. 2. Western blot analysis of NBL1 protein in PCa cell lines. NBL1 expression in DU145 and LNCaP cell lines was observed in cell lysate. In culture medium, high NBL1 expression was noted in DU145 cells (a). NBL1 expression in DU145 was suppressed by treatment with siRNA1 and siRNA2 in both cell lysate and culture medium (b).

and was detected in the prostatic ducts. Expression of NBL1 was not detected in stromal cells. Although NBL1 expression in PCa cells has some heterogeneity, NBL1 expression was detected in 1-95\% of all samples (fig. 3e-h). All prostate samples were considered NBL1 positive if any cell stained positive. These immunohistochemical results suggest that NBL1 was highly expressed in and restricted to the prostate.

Next, we compared clinicopathological parameters with NBL1 expression scores in the prostate samples (fig. 4a-d). Mean NBL1 expression score was significantly higher in $\mathrm{BPH}$ samples and $\mathrm{NP}$ adjacent to $\mathrm{PCa}$ than in PCa itself $(\mathrm{p}<0.0001)$. The mean NBL1 expression score was significantly higher in $\mathrm{PCa}$ classified as stage $\mathrm{B}$ than in PCa classified as stages $C$ and D $(p=0.0014)$ and was significantly higher in PCa with Gleason score 6 than in PCa with Gleason score $7-10(\mathrm{p}=0.0024)$. The mean NBL1 expression score was also significantly higher in PCa with PSA level $\leq 20$ than in PCa with PSA level $>20$ $(\mathrm{p}<0.0001)$.

CAST Analysis of Secreted Protein in $\mathrm{PCa}$

\section{Effect of NBL1 Inhibition on Cell Growth and} Invasive Activity of $\mathrm{PCa}$ Cells

We studied the biological role of NBL1 using the DU145 cell line because of the high expression of NBL1 in this cell line. To investigate the possible proliferative effects of NBL1 knockdown, we performed an MTT assay 4 days after siRNA transfection (fig. 4e). Cell viability was not significantly different between NBL1 siRNA-transfected DU145 and negative control siRNA-transfected DU145. Next, to determine the possible role of NBL1 in invasiveness, a transwell invasion assay was performed (fig. 4f). Invasion ability 1 day after siRNA transfection was not significantly different between NBL1 siRNAtransfected DU145 and negative control siRNA-transfected DU145.

\section{Discussion}

In the present study, we identified several genes that encode secreted proteins present in PCa and NP from CAST libraries. Quantitative RT-PCR revealed that $M S M B, N B L 1$ and $A Z G P 1$ were expressed to a much higher extent in PCa and NP than in 14 types of normal tissues. MSMB, prostatic acid phosphatase and PSA are the three most abundant proteins found in semen. It was reported that MSMB also has high specificity for the prostate and has utility as a serum biomarker for PCa [24]. In addition, a single-nucleotide polymorphism of $M S M B$ has been reported to increase the risk of developing PCa [25]. It was also reported that immunohistochemical staining of AZGP1 was a predictor of tumor recurrence and could be used as a specific serum biomarker for PCa $[26,27]$. Although we could not detect PSA in our CAST library, we think that PSA might be one of the genes difficult to ligate into the pCAST vector. In contrast, little is known about NBL1 expression in PCa. NBL1 can stimulate differentiation of neuroblastoma cells in culture in the presence of retinoic acid [30], and its growth-suppressive activity has been noted in sarcoma cells [31]. NBL1 may play an important role in preventing cells from entering into the $\mathrm{S}$ phase [32]. NBL1 mRNA was reported to be down-regulated in PCa compared with corresponding NP by quantitative RT-PCR analysis [28, 29].

Because the genes in the present study were identified by CAST analysis of PCa cell lines and quantitative RTPCR analysis of bulk PCa and NP tissues, immunohistochemical analysis was required to determine which cells expressed these genes. With this analysis, we confirmed that NBL1 was highly expressed in the epithelium of PCa

Pathobiology 2013;80:60-69 

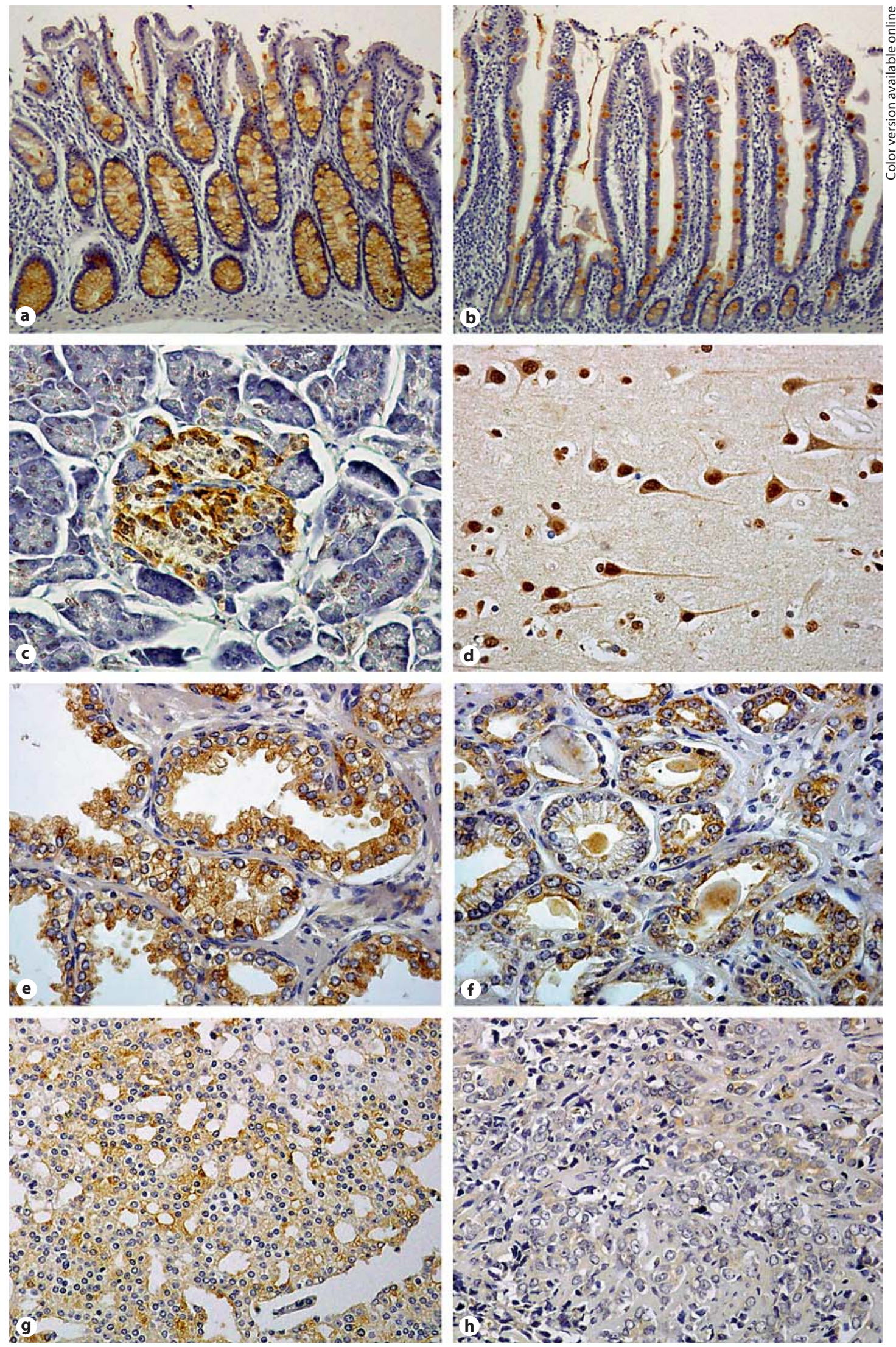

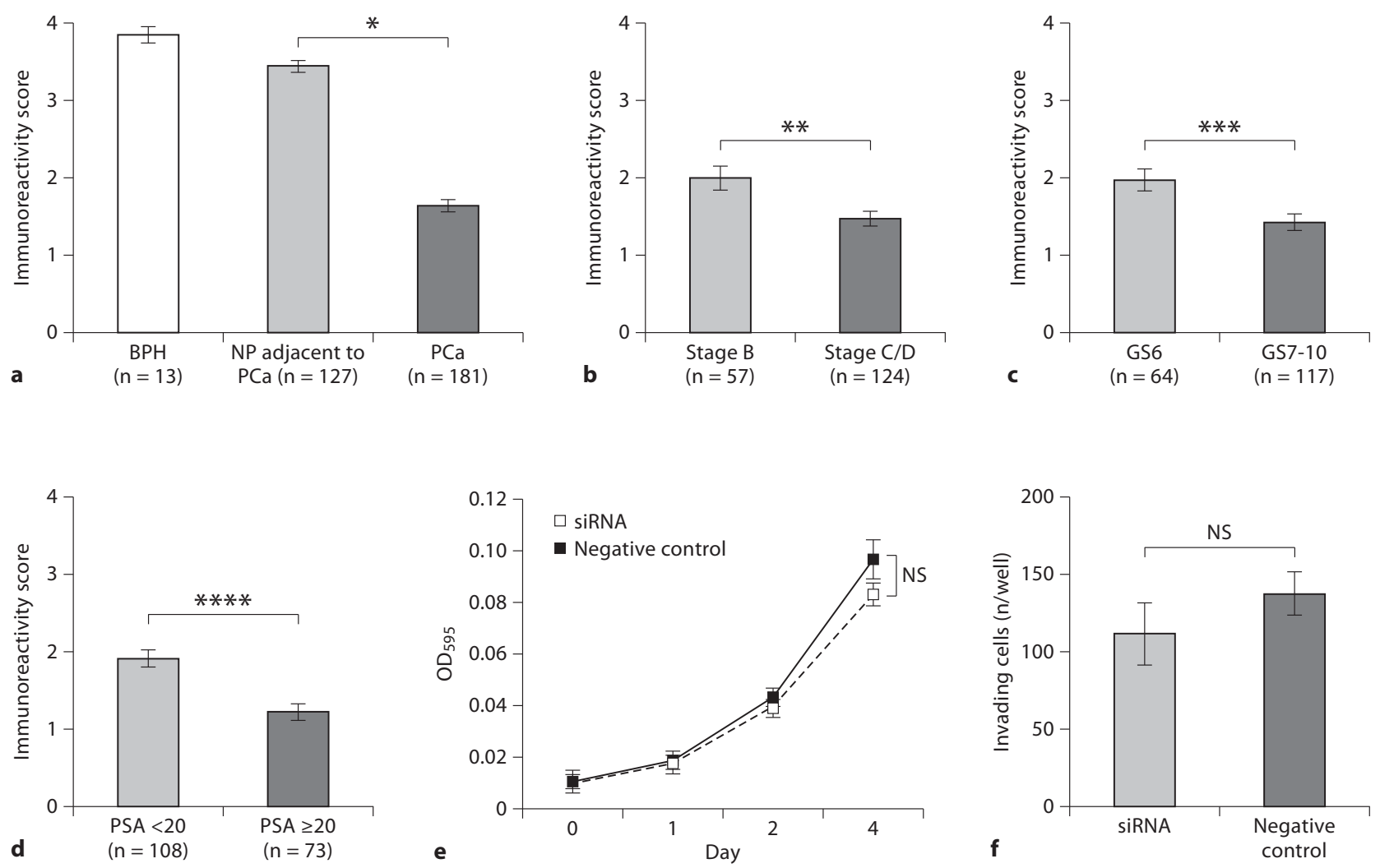

Fig. 4. NBL1 immunoreactivity scores in prostate. NBL1 expression score was higher in NP than in PCa. Means \pm SEM. BPH (a). NBL1 expression score was higher in stage $B$ than in stages $C$ and D (b). NBL1 expression score was higher in PCa with Gleason score 6 than in PCa with Gleason score 7-10 (c). NBL1 expression score was higher in PCa with PSA $\leq 20$ than in PCa with PSA $>20$

and NP. In prostate samples, NBL1 expression was high in NP and lower in PCa. Furthermore, average NBL1 expression was significantly reduced according to the progression of stage, Gleason grade and preoperative PSA value. Because NBL1 functions as a tumor suppressor gene, these results were consistent with those of previous

Fig. 3. Immunohistochemical analysis of NBL1 in non-neoplastic human tissues and prostate tissues. NBL1 expression was detected in the epithelium of the small intestine and colon, islets of the pancreas and nerve cells, respectively (a-d). NBL1 staining was observed in the cytoplasm of normal prostate epithelium and $\mathrm{PCa}$ cells with Gleason scores 3, 4 and 5, respectively. NBL1 expression was also detected in prostatic ducts $(\mathbf{e}-\mathbf{h})$. (d). e, $\mathbf{f}$ Effect of NBL1 knockdown on cell growth (e) and cell invasion (f) of DU145 cells. e Cell growth was assessed by an MTT assay 1, 2 and 4 days after seeding on 96-well plates in DU145 cells. f Invading cells were counted after 1 day. Bars and error bars, means and SD of three different experiments. NS $=$ Not significant. ${ }^{*} \mathrm{p}<0.0001 ;{ }^{* *} \mathrm{p}=0.0014 ;{ }^{* * *} \mathrm{p}=0.0024 ;{ }^{* * *} \mathrm{p}<0.0001$.

reports. NBL1 expression was detected in epithelium of the intestine, pancreatic islets and nerve cells, but was absent in other non-cancerous systemic tissues and stromal cells in adult humans. Ozaki et al. [33] examined NBL1 expression in rat tissue by Northern blot analysis and showed that NBL1 was detected in brain, intestine, kidney and lung. They did not test NBL1 expression in rat prostate, but their results were similar to those of our present study in humans.

We also confirmed with Western blot analysis that high NBL1 expression was detected in DU145 cells in culture medium. Nakamura et al. [15] also reported that NBL1 was observed in the culture medium, and the amount of NBL1 secreted from the cells was calculated to be $80 \%$ of the total NBL1 protein. Furthermore, NBL1 
expression was detected in prostatic ducts in PCa and NP. Therefore, NBL1 may be secreted into semen as well as MSMB, prostatic acid phosphatase and PSA. PSA is produced by secretory epithelial cells in the acini and ducts, and it is secreted directly into the lumen. A characteristic feature of $\mathrm{PCa}$ is disruption of the basal cell layer and basement membrane, and this loss of the normal glandular architecture appears to allow PSA increased direct access to the peripheral circulation. PSA is normally found at lower levels in paraurethral and perianal glands, apocrine sweat glands, breast, thyroid and placenta, but these sites do not normally contribute measurable levels of PSA to the circulation [34]. Therefore, in spite of the fact that PSA expression is also higher in NP than in PCa in immunohistochemical analysis, serum PSA is increased in patients with PCa. Although establishment of an enzyme-linked immunosorbent assay system for serum samples is needed to clarify whether NBL1 can serve as a serum marker for detecting and monitoring PCa, we believe that NBL1 might be useful as a serum biomarker for PCa. NBL1 might be beneficial in addition to PSA in situations where PSA is less useful, such as in patients with low PSA level or castration-refractory disease.

The regulation of NBL1 is poorly understood, especially in PCa. Because NBL1 is thought to be a tumor suppressor gene, it is thought that NBL1 in PCa has mutations, deletions and methylation. Ozaki et al. [35] reported that two transcription sites were present in the rat NBL1 gene, suggesting the possibility of transcriptional regulation of NBL1. Further studies will clarify how NBL1 is regulated and whether androgen and the andro- gen receptor axis regulate NBL1. The biological function of the NBL1 protein is also poorly understood in PCa. NBL1 expression correlated with PCa progression, but NBL1 knockdown did not reduce viability and invasiveness relative to the negative control. Because NBL1 was also reported to act as a bone morphogenetic protein (BMP) antagonist by binding to BMPs [36] and BMPs are known to participate in the progression of $\mathrm{PCa}[37,38]$, we speculate that interaction between NBL1 and BMP may play a more important role during growth and development in PCa than in cell cycle inhibition.

In summary, the present study yielded a list of genes that encode secreted proteins present in $\mathrm{PCa}$ and NP from CAST analysis. NBL1 expression is narrowly restricted to the prostate and is higher in NP than in PCa. Underexpression of NBL1 is associated with tumor progression. NBL1 has high potential as a biomarker of $\mathrm{PCa}$ and its progression.

\section{Acknowledgments}

We thank Mr. Shinichi Norimura for his excellent technical assistance and advice. This work was carried out with the kind cooperation of the Research Center for Molecular Medicine, Faculty of Medicine, Hiroshima University. We thank the Analysis Center of Life Science, Hiroshima University, for the provision of their facilities. This work was supported in part by Grants-in-Aid for Cancer Research from the Ministry of Education, Culture, Science, Sports and Technology of Japan; in part by a Grant-in-Aid for the Third Comprehensive 10-Year Strategy for Cancer Control and for Cancer Research from the Ministry of Health, Labor and Welfare of Japan, and in part by a grant (07-23911) from the Princess Takamatsu Cancer Research Fund.

\section{References}

1 Yasui W, Oue N, Kitadai Y, Nakayama H: Recent advances in molecular pathobiology of gastric carcinoma; in Kaminishi M, Takubo K, Mafune K (eds): The Diversity of Gastric Carcinoma Pathogenesis: Diagnosis, and Therapy. Tokyo, Springer, 2005, pp 51-71.

$\checkmark 2$ Yasui W, Oue N, Ito R, Kuraoka K, Nakayama H: Search for new biomarkers of gastric cancer through serial analysis of gene expression and its clinical implications. Cancer Sci 2004;95:385-392.

$\checkmark 3$ Hayashi T, Oue N, Sakamoto N, et al: Identification of transmembrane protein in prostate cancer by the Escherichia coli ampicillin secretion trap: expression of CDON is involved in tumor cell growth and invasion. Pathobiology 2011;78:277-284.
4 Anami K, Oue N, Noguchi T, et al: Search for transmembrane protein in gastric cancer by the Escherichia coli ampicillin secretion trap: expression of DSC2 in gastric cancer with intestinal phenotype. J Pathol 2010;221:275284.

5 Sirovich BE, Schwartz LM, Woloshin S: Screening men for prostate and colorectal cancer in the United States: does practice reflect the evidence? JAMA 2003;289:14141420.

6 Boyle P: Screening for prostate cancer: have you had your cholesterol measured? BJU Int 2003;92:191-199.

7 Carter HB: Prostate cancers in men with low PSA levels - must we find them? N Engl J Med 2004;350:2292-2294.
8 Gambert SR: Prostate cancer. When to offer screening in the primary care setting. Geriatrics 2001;56:22-26.

$\checkmark 9$ Edwards S, Campbell C, Flohr P, et al: Expression analysis onto microarrays of randomly selected cDNA clones highlights HOXB13 as a marker of human prostate cancer. Br J Cancer 2005;92:376-381.

10 Oefelein MG, Resnick MI: Effective testosterone suppression for patients with prostate cancer: is there a best castration? Urology 2003;62:207-213.

11 Chang SS, Kibel AS: The role of systemic cytotoxic therapy for prostate cancer. BJU Int 2009;103:8-17. 
$\longrightarrow 12$ Ferguson DA, Muenster MR, Zang Q, et al: Selective identification of secreted and transmembrane breast cancer markers using Escherichia coli ampicillin secretion trap. Cancer Res 2005;65:8209-8217.

13 von Heijne G: A new method for predicting signal sequence cleavage sites. Nucleic Acids Res 1986;14:4683-4690.

-14 Kadonaga JT, Gautier AE, Straus DR, Charles $\mathrm{AD}$, Edge MD, Knowles JR: The role of the beta-lactamase signal sequence in the secretion of proteins by Escherichia coli. J Biol Chem 1984;259:2149-2154.

-15 Nakamura Y, Ozaki T, Nakagawara A, Sakiyama S: A product of DAN, a novel candidate tumour suppressor gene, is secreted into culture medium and suppresses DNA synthesis. Eur J Cancer 1997;33:1986-1990.

16 Sobin LH, Wittekind CH (eds): TNM Classification of Malignant Tumors, ed 6. New York, Wiley-Liss, 2002, pp 184-187.

17 Eble JN, Sauter G, Epstein JI, et al: World Health Organization Classification of $\mathrm{Tu}$ mours. Pathology and Genetics of Tumours of the Urinary System and Male Genital Organs. Lyon, IARC Press, 2004.

18 Kondo T, Oue N, Yoshida K, et al: Expression of POT1 is associated with tumor stage and telomere length in gastric carcinoma. Cancer Res 2004;64:523-529.

$\checkmark 19$ Gibson UE, Heid CA, Williams PM: A novel method for real time quantitative RT-PCR. Genome Res 1996;6:995-1001.

20 Yasui W, Ayhan A, Kitadai Y, et al: Increased expression of p34cdc2 and its kinase activity in human gastric and colonic carcinomas. Int J Cancer 1993;53:36-41.

21 Ohtori S, Yamamoto T, Ino H, et al: Differential screening-selected gene aberrative in neuroblastoma protein modulates inflammatory pain in the spinal dorsal horn. Neuroscience 2002;110:579-586.
22 Sakamoto N, Oue N, Noguchi T, et al: Serial analysis of gene expression of esophageal squamous cell carcinoma: ADAMTS16 is upregulated in esophageal squamous cell carcinoma. Cancer Sci 2010;101:1038-1044.

23 Alley MC, Scudiero DA, Monks A, et al: Feasibility of drug screening with panels of human tumor cell lines using a microculture tetrazolium assay. Cancer Res 1988;48:589601.

24 Whitaker HC, Warren AY, Eeles R, Kote-Jarai Z, Neal DE: The potential value of microseminoprotein- $\beta$ as a prostate cancer biomarker and therapeutic target. Prostate 2010;70:333-340.

25 Xu B, Wang J, Tong N, et al: A functional polymorphism in MSMB gene promoter is associated with prostate cancer risk and serum MSMB expression. Prostate 2010;70: 1146-1152.

26 Henshall SM, Horvath LG, Quinn DI, et al: Zinc- $\alpha_{2}$-glycoprotein expression as a predictor of metastatic prostate cancer following radical prostatectomy. J Natl Cancer Inst 2006;98:1420-1424.

27 Lapointe J, Li C, Higgins JP, et al: Gene expression profiling identifies clinically relevant subtypes of prostate cancer. Proc Natl Acad Sci USA 2004;101:811-816.

28 Asmann YW, Kosari F, Wang K, Cheville JC, Vasmatzis G: Identification of differentially expressed genes in normal and malignant prostate by electronic profiling of expressed sequence tags. Cancer Res 2002;62:33083314.

-29 Shaikhibrahim Z, Lindstrot A, Buettner R, Wernert N: Analysis of laser-microdissected prostate cancer tissues reveals potential tumor markers. Int J Mol Med 2011;28:605611.
30 Nakamura Y, Ozaki T, Ichimiya S, Nakagawara A, Sakiyama S: Ectopic expression of DAN enhances the retinoic acid-induced neuronal differentiation in human neuroblastoma cell lines. Biochem Biophys Res Commun 1998;243:722-726.

- 31 Hanaoka E, Ozaki T, Nakamura Y, Moriya $\mathrm{H}$, Nakagawara A, Sakiyama S: Overexpression of DAN causes a growth suppression in p53-deficient SAOS-2 cells. Biochem Biophys Res Commun 2000;278:20-26.

32 Ozaki T, Nakayama Y, Enomoto H, Hirose M, Sakiyama S: Overexpression of DAN gene product in normal rat fibroblasts causes a retardation of the entry into the S phase. Cancer Res 1995;55:895-900.

-33 Ozaki T, Sakiyama S: Molecular cloning and characterization of a cDNA showing negative regulation in v-src-transformed $3 \mathrm{Y} 1$ rat fibroblasts. Proc Natl Acad Sci USA 1993;90: 2593-2597.

34 Balk SP, Ko YJ, Bubley GJ: Biology of prostate-specific antigen. J Clin Oncol 2003;21: 383-391.

35 Ozaki T, Enomoto H, Nakamura Y, et al: The genomic analysis of human DAN gene. DNA Cell Biol 1997;16:1031-1039.

36 Kim AS, Pleasure SJ: Expression of the BMP antagonist Dan during murine forebrain development. Brain Res Dev Brain Res 2003; 145:159-162.

37 Hamdy F, Autzen P, Robinson MC, Horne $\mathrm{CH}$, Neal DE, Robson CN: Immunolocalization and messenger RNA expression of bone morphogenetic protein- 6 in human benign and malignant prostate tissue. Cancer Res 1997;57:4427-4431.

38 Dai J, Keller J, Zhang J, Lu Y, Yao Z, Keller ET: Bone morphogenetic protein- 6 promotes osteoblastic prostate cancer bone metastases through a dual mechanism. Cancer Res 2005;65:8274-8285. 